\title{
An adaptive meshfree method for phase-field models of biomembranes. Part II: a Lagrangian approach for membranes in viscous fluids
}

\author{
C. Peco, A. Rosolen ${ }^{\text {ts }}$ and M. Arroyo* \\ LaCàN, Universitat Politècnica de Catalunya-BarcelonaTech (UPC), Barcelona 08034, Spain
}

\begin{abstract}
We present a Lagrangian phase-field method to study the low Reynolds number dynamics of vesicles embedded in a viscous fluid. In contrast to previous approaches, where the field variables are the phase-field and the fluid velocity, here we exploit the fact that the phase-field tracks a material interface to reformulate the problem in terms of the Lagrangian motion of a background medium, containing both the biomembrane and the fluid. We discretize the equations in space with maximum-entropy approximants, carefully shown to perform well in phase-field models of biomembranes in a companion paper. The proposed formulation is variational, lending itself to implicit time-stepping algorithms base on minimization of a time-incremental energy, which are automatically nonlinearly stable. The proposed method deals with two of the major challenges in the numerical treatment of coupled fluid/phase-field models of biomembranes, namely the adaptivity of the grid to resolve the sharp features of the phase-field, and the stiffness of the equations, leading to very small time-steps. In our method, local refinement follows the features of the phase-field as both are advected by the Lagrangian motion, and large time-steps can be robustly chosen in the variational time-stepping algorithm, which also lends itself to time adaptivity. The method is presented in the axisymmetric setting, but it can be directly extended to $3 \mathrm{D}$.
\end{abstract}

Keywords: phase field models, biomembranes, vesicles, meshfree methods, variational methods, adaptivity

\section{Introduction}

Biomembranes self-assemble in a fluid, and often, the fluid mechanics are important in their dynamical behavior. Examples include the dynamics of vesicles in shear flows (see, e.g. [1, 2, $3,4]$ ), or the relaxation dynamics of membrane structures brought out-of-equilibrium [5, 6]. Describing explicitly the fluid surrounding biomembranes may also be useful in studying the interactions between membranes and other structures [7]. Here, we consider the simplest, yet very common and useful model of a biomembrane: an inextensible interface with curvature elasticity, given by the Helfrich energy. We ignore here the bilayer architecture, the monolayer

\footnotetext{
${ }^{\text {th }}$ Current address: Institute for Soldier Nanotechnologies, MIT, Cambridge MA, USA

*Correspondence to: marino.arroyo@upc.edu
} 
extensibility, the surface viscosity, and the inter-monolayer friction, which can be important in some situations [8]. Our goal here is to develop a robust and efficient computational technique for biomembranes embedded in a viscous fluid, capable of handling arbitrarily large shape changes and the associated flows. We resort to phase-field models of biomembranes, and propose a nonconventional discretization of the membrane-fluid system. In a companion paper [9], we have shown that high-order phase-field models of biomembranes can be accurately approximated in a direct Galerkin approach with the maximum-entropy meshfree approximants [10], in an adaptive, accurate and efficient way. Here, we elaborate a Lagrangian method for the dynamics of vesicles embedded in a viscous fluid, which builds on the meshfree approximation of the phasefield equations. The proposed method shares common features with the optimal mass transport (OTM) method presented in [11].

\section{Background}

A number of models and numerical approaches have been proposed to analyze the hydrodynamics of fluid membranes in a viscous fluid. These include a mesoscopic model, combining a particle-based method for the fluid and a dynamically triangulated surface model for the membrane [3], which has been put forth to study the effect of membrane viscosity and thermal fluctuations in the dynamical behavior of vesicles in simple shear flow, as well as the behavior of vesicles and red blood cells in microcapillaries [12]. Other methods rely on conventional

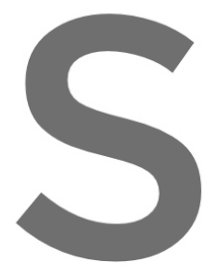
continuum mechan a Lagrangian form ternative sharp-intert spherical harmonic: integral method for many interacting vesicles. Immersed boundary
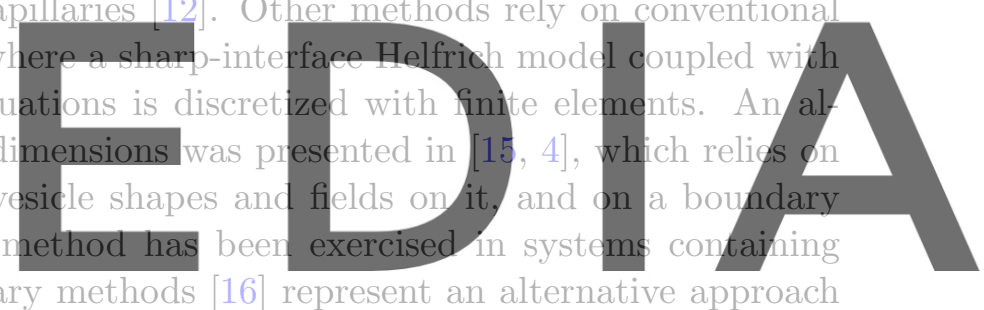

Register for handle fluid-structure interaction, maintaining the Eulerian framework for the fluid media free at https/.WWWw. sci.pedia.com to download the version without the watermark have been applied to understand the hydrodynamic effects on fluid vesicles and biomembranes in $[17,18]$. Phase-field models of vesicles [19] have also been coupled with the ambient hydrodynamics $[20,21]$, through an Eulerian description of the fluid with a source term of membrane elastic forces, and a transport equation to advect the phase-field representing the membrane. Alternative phase-field approaches to vesicles in a flow have been proposed in [22, 23], where the local area inextensibility was also accounted for, and in [24].

Phase-field models offer advantages when compared to sharp-interface models, in that they provide unified treatment of interface tracking and surface mechanics with a single partial differential equation (PDE) governing the phase-field. Phase-field approaches do not suffer from severe mesh distortions, and can easily deal with large deformations and even topology changes $[22,25]$ without demanding specific reparametrization techniques [13] or control of the tangential motions of the nodes [26]. In contrast, phase-field models are encoded by nonlinear PDEs, often high-order, which develop sharp features, and therefore present computational challenges. In phase-field models of biomembranes, an artificial length-scale $\epsilon$ governing the width of the smeared interface is introduced, and the sharp-interface limit is recovered as $\epsilon \rightarrow 0$ [19, 22, 27]. For phase-field models to accurately represent the sharp-interface limit, $\epsilon$ needs to be much 
smaller than other relevant dimensions in the problem. Furthermore, this length-scale needs to be resolved by the computational grids, typically leading to expensive calculations. From a practical viewpoint, the high computational cost, associated with increasing the dimension of the problem and having to resolve numerically the (small) thickness of the smeared interface, can be outweighed by their simplicity, making them amenable to scalable parallel implementations. Besides, the computational cost can be considerably mitigated with spacial adaptivity [28, 29].

\section{The proposed method}

In previous phase-field approaches to the ambient hydrodynamics-biomembrane mechanics, the problem is formulated in a Eulerian frame, as a coupled system combining the fluid flow equations with a source term coming from curvature elasticity forces and the advection of the phase-field with the flow, in which the phase-field and the fluid velocity (and pressure) are the unknowns $[22,25]$. In such approaches, adaptive strategies, not proposed so far, would require cumbersome grid projection steps. Since in the present situation the phase-field tracks a material interface, here we view the phase-field as a material property, and formulate a Lagrangian description of the problem in which the unknown is the Lagrangian motion of the background medium, containing both the fluid and the smeared interface (see Fig. 1 for an illustration). See [30] for a related approach. We particularize the model with the phase-field approach proposed by [19], and since biomembranes often operate in the limit of vanishing Reynolds number

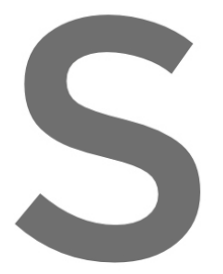
describe the hydrodynamics with Stoke equations.
With the Lagrangian viewpoint, when discretized in sp
model becomes a nonlinear dissipative particle system, driven b
by a viscous force ddmitting a dissipation potential, whose
subject to area and volume constraints. With the sane sp

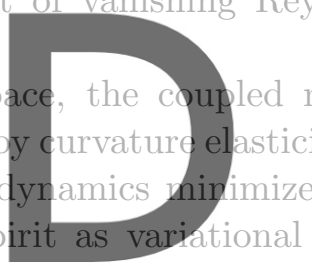

Hamiltonian systems [32], we choose to discretize in time the action, and then derive by con-

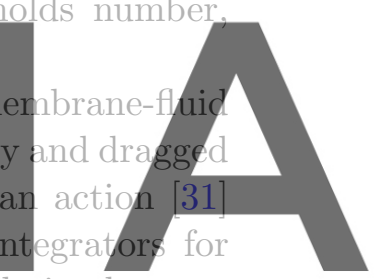

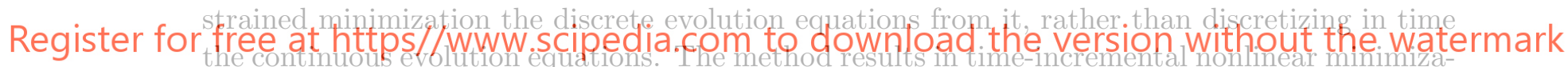

tion problems, as in modern treatments of dissipative processes in materials science following the seminal work in [33]. As a consequence, it is possible to overcome the stiffness of the dynamics (given by the fourth-order nature of the PDE) and take robustly large time-steps. Furthermore, the algorithm is automatically nonlinearly stable as the energy monotonically decreases.

If the initial grid adapts to the features of the phase-field, adaptivity is advected by the Lagrangian map, and therefore local refinement along the dynamics is accomplished for free (see Fig. 1). The Lagrangian framework allows us to pull-back the successive states of the system to a reference configuration. Thus, we avoid the calculation of the meshfree basis functions in every step of the evolution. It has been shown that the meshfree method considered here can withstand significant deformation before the discretized deformation mapping ceases to be injective (i.e. the Jacobian determinant becomes negative at a quadrature point) [10]. However, we avoid coming close to this limit, which degrades the accuracy of the approximation, by reconnecting the nodes, recomputing the basis functions, and resetting the reference configuration periodically along the simulation. These reconnection steps are seamless, as detailed later: they do not involve remeshing, recomputing the background grid for quadrature, field projections, nor do they alter in any way the variational structure of the discrete equations, e.g. the nonlinear stability of the 


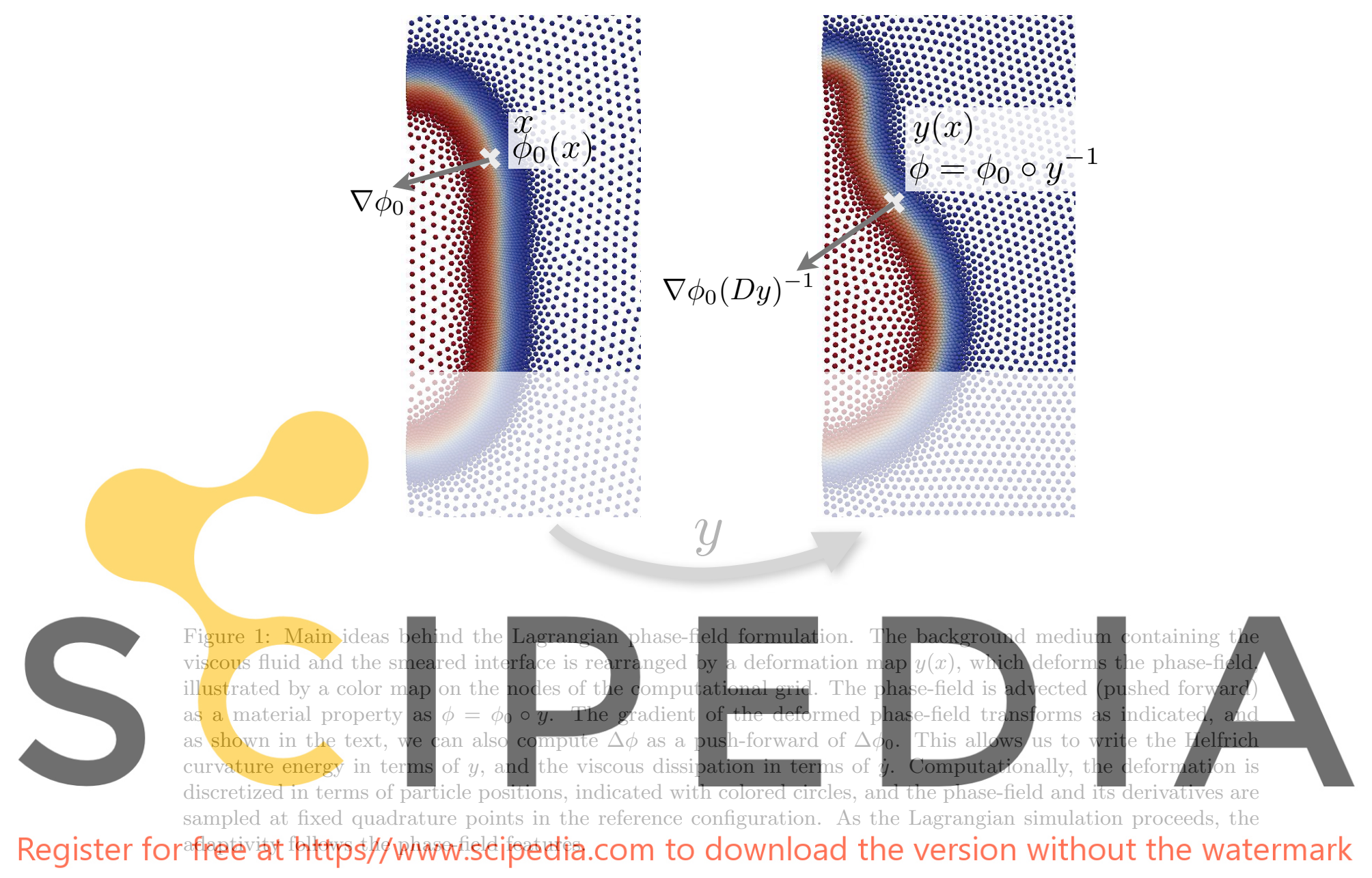

dynamics. In situations involving extreme Lagrangian deformations, particles may accumulate or cover insufficiently parts of the domain. In such cases, a full re-meshing and field projections are required. We did not find the need to doing this, except in the example depicted in Figure 6.

From a purely numerical viewpoint, as exemplified later, accounting for the ambient fluid can help in devising adaptive strategies in space, resolving with detail the sharp and moving features of the phase-field, even if we are only interested in equilibria. Indeed, vesicles are prone to buckling events, i.e. large shape transformations under small changes of the enclosed volume or the spontaneous curvature as the system transitions between different metastable equilibrium branches. Consequently, if the grid is locally adapted to a given conformation [28] and the control parameters are slightly perturbed, the new energy minimizer may not be well described by the current mesh, strongly biased by the previous minimizer. This poses a serious challenge to adaptive phase-field methods based on free energy minimization. In contrast with possibly discontinuous equilibrium paths, dynamics are always continuous, making it possible to gradually adapt the resolution to the phase-field. Gradient flow dynamics, even without a 
clear physical meaning $[34,27,13]$, have been used to numerically obtain equilibrium shapes, and the adaptive method we proposed here can be used in this vein.

The structure of this paper is as follows. In Section 2, we derive the Lagrangian formulation for the phase-field membrane embedded in a viscous fluid, and obtain variationally the governing equations of the coupled dynamics. In Section 3, we propose the space and time discretization. In Section 4, we illustrate the method with several examples. Finally, we collect conclusions in Section 5 .

\section{Lagrangian phase-field formulation for biomembranes in a viscous fluid}

\subsection{Lagrangian form of the phase-field model}

The formulation we present here is three-dimensional, and the particularization to axisymmetry is given in Appendix A. Consider a fixed fluid domain $\Omega$, containing a fluid membrane described at time $t=0$ by a phase-field $\phi_{0}(x)$. Such initial phase-field may result from an equilibrium calculation. Consider now a motion of the background continuum medium containing both the fluid and the smeared interface, i.e. a smooth bijective mapping on $\Omega$ at each instant of time, $y_{t}(x)[35,36]$. Viewing the phase-field as a material property, attached to the material particles, it is pushed forward by the motion following
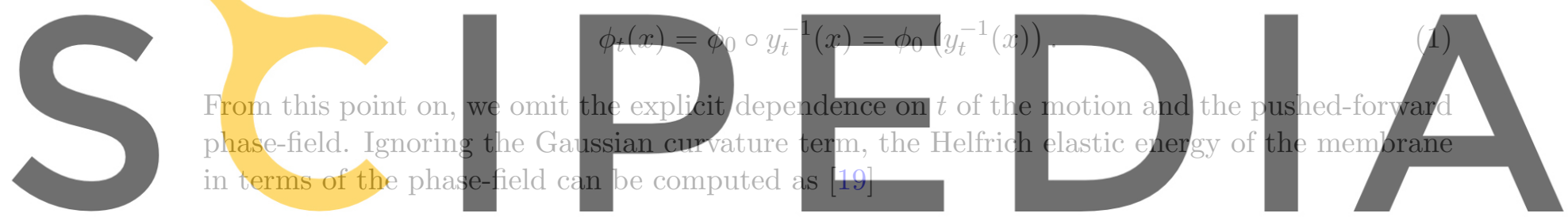

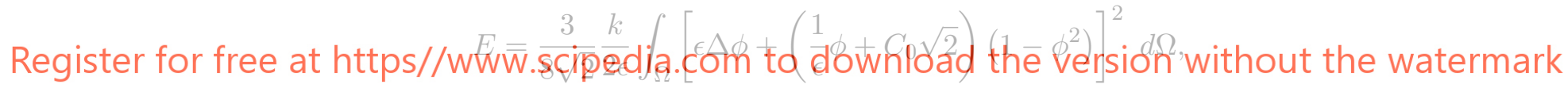

where $k$ is the bending stiffness of the bilayer, and $\epsilon$ is a regularization parameter controlling the width of the smeared interface. The enclosed volume and surface are can be computed as

$$
V=\frac{1}{2}\left(\operatorname{Vol}(\Omega)+\int_{\Omega} \phi d \Omega\right)
$$

and

$$
A=\frac{3}{2 \sqrt{2}} \int_{\Omega}\left[\frac{\epsilon}{2}|\nabla \phi|^{2}+\frac{1}{4 \epsilon}\left(\phi^{2}-1\right)^{2}\right] d \Omega
$$

To compute the spacial derivatives of the phase-field, we recall Eq. (1) and the inverse function theorem to obtain

$$
\nabla \phi=\left(\nabla \phi_{0} F^{-1}\right) \circ y^{-1}
$$

where $F=D y$ is the deformation gradient, or $F_{i I}=\partial_{I} y_{i}$, where upper-case subindices denote indices or partial differentiation with respect to material (Lagrangian) coordinates, i.e. " $x$ ", while lower-case subindices refer to spacial (Eulerian) coordinates, i.e. " $y$ ". To compute the Laplacian of the pushed-forward phase-field, we resort to indicial notation and omit the composition with 
the deformation map or its inverse as it can be inferred from the context. From the relation $\partial_{i} \phi=\partial_{I} \phi_{0} F_{I i}^{-1}$ we have

$$
\partial_{i j}^{2} \phi \circ y=\partial_{I J}^{2} \phi_{0} F_{I i}^{-1} F_{J j}^{-1}+\partial_{I} \phi_{0} \partial_{j} F_{I i}^{-1}
$$

Now, from $F_{I k}^{-1} F_{k J}=\delta_{I J}$, we obtain

$$
\partial_{j} F_{I i}^{-1}=-F_{I n}^{-1} F_{J i}^{-1} F_{K j}^{-1} \partial_{K} F_{n J}=-F_{I n}^{-1} F_{J i}^{-1} F_{K j}^{-1} \partial_{J K}^{2} y_{n} .
$$

In particular, we have

$$
\Delta \phi \circ y=\partial_{I J}^{2} \phi_{0} F_{I i}^{-1} F_{J i}^{-1}+\partial_{I} \phi_{0} \partial_{i} F_{I i}^{-1} .
$$

Thus, inserting these Eqs. $(2,4)$ into the above functionals and pulling-back the integration by the deformation map to the Lagrangian domain, the elastic energy, enclosed volume, and surface area can be interpreted as functions of the deformation mapping alone, depending parametrically on the initial phase-field:

$$
E[y]=\frac{3}{8 \sqrt{2}} \frac{k}{2 \epsilon} \int_{\Omega}\left[\epsilon \Delta \phi \circ y+\left(\frac{1}{\epsilon} \phi_{0}+C_{0} \sqrt{2}\right)\left(1-\phi_{0}^{2}\right)\right]^{2} \operatorname{det}(F) d \Omega,
$$
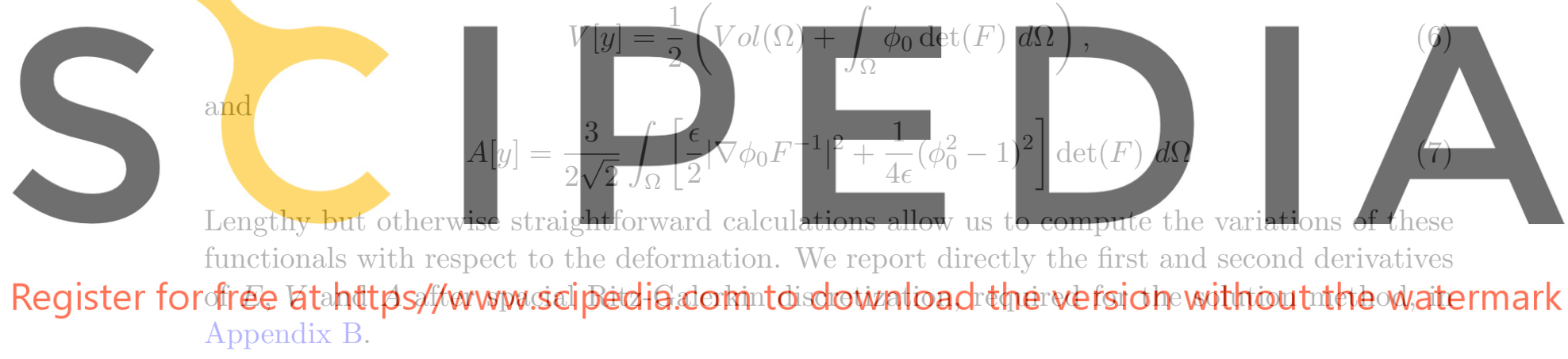

\subsection{Lagrangian form of the fluid dissipation potential}

To simplify the exposition of the method, we consider the Stokes equations for a slightly compressible fluid, i.e. a penalized formulation of the incompressible Stokes equations. The numerical treatment of the incompressible case with a stabilized maximum-entropy meshfree method is straightforward [37], but would distract from the main ideas of the present work.

Following standard continuum mechanics definitions, the Eulerian velocity field can be computed as

$$
v=\partial_{t} y \circ y^{-1}
$$

Consequently, the velocity gradient tensor can be written as

$$
\nabla v \circ y=\dot{F} F^{-1},
$$

where $\dot{F}_{i I}=\partial_{I} \partial_{t} y_{i}$, and the rate-of-deformation tensor in the Lagrangian domain as

$$
d \circ y=\frac{1}{2}\left(\dot{F} F^{-1}+F^{-T} \dot{F}^{T}\right) .
$$


The Rayleigh dissipation potential for a compressible Newtonian fluid can therefore be written as $[38]$

$$
\begin{aligned}
\operatorname{Diss}\left[\partial_{t} y ; y\right] & =\mu \int_{\Omega} d: d d \Omega+\frac{\lambda}{2} \int_{\Omega}(\operatorname{div} v)^{2} d \Omega \\
& =\frac{\mu}{4} \int_{\Omega}\left|\dot{F} F^{-1}+F^{-T} \dot{F}^{T}\right|^{2}(\operatorname{det} F) d \Omega+\frac{\lambda}{2} \int_{\Omega}\left[\operatorname{trace}\left(\dot{F} F^{-1}\right)\right]^{2}(\operatorname{det} F) d \Omega .
\end{aligned}
$$

where $\mu$ is the shear viscosity of the fluid, and by $\operatorname{Diss}\left[\partial_{t} y ; y\right]$ we highlight the parametric dependence of the functional on the current deformation $y$. The coefficient $\lambda$ can be interpreted here as a penalty parameter enforcing incompressibility approximately. For an incompressible Newtonian fluid, the second term above is replaced by the constraint

$$
\operatorname{tr}\left(\dot{F} F^{-1}\right)=0,
$$

\section{the linearization of the condition $\operatorname{det} F=1$. \\ 2.3. Governing equations}

The dynamics of the system can be obtained by minimizing the Rayleigh dissipation potential plus the rate of change of the elastic energy with respect to the variables expressing the rate of
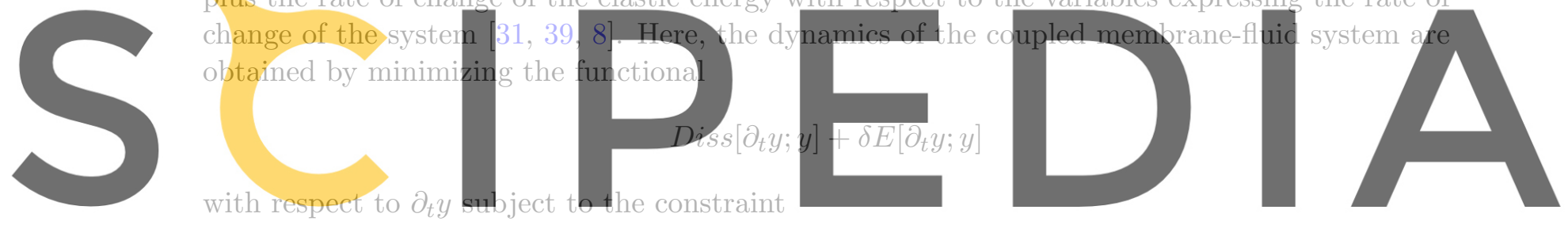

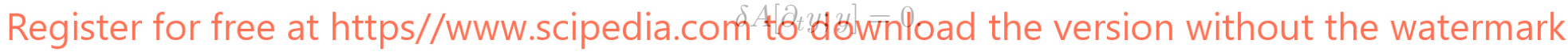

To control the enclosed volume explicitly, without relying on the fluid (quasi)-incompressiblity, the following constraint can be added

$$
\delta V\left[\partial_{t} y ; y\right]=0 .
$$

We note that this formulation enforces the global area preservation along the dynamics, while physically, local area preservation throughout the membrane is more meaningful, see $[4,8]$ and [23] for the phase-field modeling the local constraint. Again, for the sake of clarity, we stick here to the global constraint as in [21].

\section{Discrete equations}

\subsection{Space discretization: function approximation and quadrature}

We start from a node set $X=\left\{x^{1}, x^{2}, \ldots, x^{N}\right\}$ adapted to the reference phase-field $\phi_{0}$, and define the associated local maximum-entropy basis functions $p_{a}(x), a=1, \ldots, N$, for a given aspect ratio parameter $\gamma[40,9]$. We also consider a set of quadrature points $\hat{X}=\left\{\hat{x}^{1}, \hat{x}^{2}, \ldots, \hat{x}^{Q}\right\}$ and the associated quadrature weights $W=\left\{w^{1}, w^{2}, \ldots, w^{Q}\right\}$, obtained for instance from a 
triangulation of the node set $X$. The quadrature points and weights are only set up once in the calculation, and are subsequently transported by the motion. They are not altered by the node reconnection steps, see below. The gradient and the Hessian of the reference phase-field can be obtained through interpolation with the smooth meshfree basis functions:

$$
\phi_{0}(x)=\sum_{a} p_{a}(x) \phi_{0}^{a}, \quad \partial_{I} \phi_{0}(x)=\sum_{a} \partial_{I} p_{a}(x) \phi_{0}^{a}, \quad \partial_{I J}^{2} \phi_{0}(x)=\sum_{a} \partial_{I J}^{2} p_{a}(x) \phi_{0}^{a},
$$

which only need to be evaluated at the quadrature points in $\hat{X}$ to yield the values $\phi_{0}^{\alpha}, \partial_{I} \phi_{0}^{\alpha}$, and $\partial_{I J}^{2} \phi_{0}^{\alpha}$ for $\alpha=1,2, \ldots, Q$. If computer memory is not an issue, these objects only need to be evaluated once at the beginning of a simulation. The motion is represented numerically as

$$
y_{t}(x)=\sum_{a} p_{a}(x) y^{a}(t) .
$$

At the initial instant we have $y^{a}(0)=x^{a}$, and as a result of the linear consistency of the approximants, $y_{0}(x)=x$. Furthermore, we have

$$
\partial_{t} y=\sum_{a} p_{a}(x) \dot{y}^{a}, \quad F_{i I}=\sum_{a} \partial_{I} p_{a}(x) y_{i}^{a}, \quad \partial_{J K}^{2} y_{n}=\sum_{a} \partial_{J K} p_{a}(x) y_{n}^{a}
$$
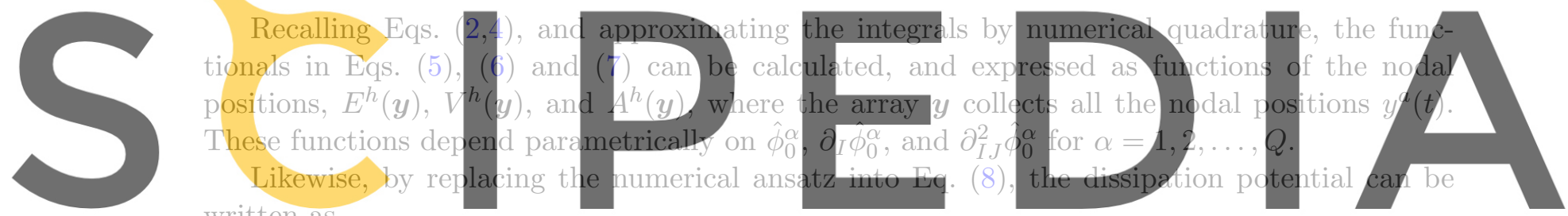

written as

\section{$\operatorname{Diss}^{h}(\dot{y} ; y)=\frac{1}{2}\left[\mu K_{a i h j}^{\mu}(y)+\lambda K_{a i b j}^{\lambda}(y)\right] \dot{y}_{i}^{a} \dot{y}_{\dot{b}}^{b}$}

Register for free at https//www.scipedia.com to download the version without the watermark where

$$
K_{a i, b j}^{\mu}(\boldsymbol{y})=\int_{\Omega}\left(\delta_{i j} F_{I k}^{-1} \partial_{I} p_{a} F_{J k}^{-1} \partial_{J p_{b}}+F_{I j}^{-1} \partial_{I} p_{a} F_{J i}^{-1} \partial_{J} p_{b}\right)(\operatorname{det} F) d \Omega
$$

and

$$
K_{a i, b j}^{\lambda}(\boldsymbol{y})=\int_{\Omega} F_{I i}^{-1} \partial_{I} p_{a} F_{J j}^{-1} \partial_{J} p_{b}(\operatorname{det} F) d \Omega .
$$

Then, the dynamics of the resulting nonlinear dissipative particle system follow from minimizing

$$
\frac{1}{2} \dot{\boldsymbol{y}}^{T} \boldsymbol{K}(\boldsymbol{y}) \dot{\boldsymbol{y}}-\boldsymbol{f}_{E}(\boldsymbol{y})^{T} \dot{\boldsymbol{y}}
$$

with respect to $\dot{\boldsymbol{y}}$ subject to

$$
\left[\nabla A^{h}(\boldsymbol{y})\right]^{T} \dot{y}=0
$$

where elastic forces are defined as $\boldsymbol{f}_{E}(\boldsymbol{y})=-\nabla E^{h}(\boldsymbol{y})$ and we note that by the chain rule $\dot{E}=-\boldsymbol{f}_{E}(\boldsymbol{y})^{T} \dot{\boldsymbol{y}}$. To account for the constraint, we write the Lagrangian

$$
\mathcal{L}(\dot{\boldsymbol{y}}, \sigma ; \boldsymbol{y})=\frac{1}{2} \dot{\boldsymbol{y}}^{T} \boldsymbol{K}(\boldsymbol{y}) \dot{\boldsymbol{y}}-\boldsymbol{f}_{E}(\boldsymbol{y})^{T} \dot{\boldsymbol{y}}+\sigma\left[\nabla A^{h}(\boldsymbol{y})\right]^{T} \dot{\boldsymbol{y}}
$$


where $\sigma$ is the membrane tension, which leads to the system

$$
\left[\begin{array}{cc}
\boldsymbol{K}(\boldsymbol{y}) & \nabla A^{h}(\boldsymbol{y}) \\
{\left[\nabla A^{h}(\boldsymbol{y})\right]^{T}} & 0
\end{array}\right]\left(\begin{array}{c}
\dot{\boldsymbol{y}} \\
\sigma
\end{array}\right)=\left(\begin{array}{c}
\boldsymbol{f}_{E}(\boldsymbol{y}) \\
0
\end{array}\right) .
$$

This system of nonlinear differential algebraic equations can be solved with standard algorithms. The system is stiff because of the nature of the curvature energy, and because of the presence of constraints. We find that standard numerical packages have serious difficulties in dealing with these equations, and require very small time-steps when the system is significantly out of equilibrium. Instead, we develop next variational time-incremental integrators, which can robustly deal with large time-steps.

\subsection{Variational time discretization}

The time-discretization in the previous section is performed on time-continuous evolution equations derived from minimizing an action subject to constraints. Here, we adopt an alternative viewpoint, by first discretizing in time the action, and then minimizing the time-discrete action with respect to the configuration of the system at time-step $n+1$.

\section{Let us consider the simplest finite difference approximations for the rate of change of the} nodal positions
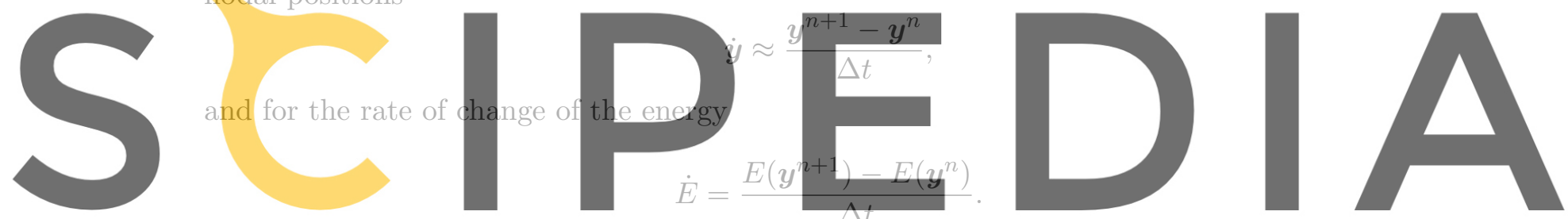

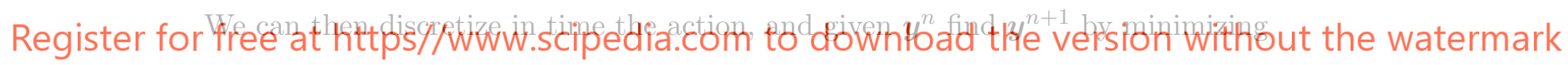

$$
\frac{1}{2}\left(y-y^{n}\right)^{T} K\left(y^{n}\right)\left(y-y^{n}\right)+\Delta t E(y)
$$

with respect to $\boldsymbol{y}$, subject to

$$
A^{h}(\boldsymbol{y})=A_{0},
$$

where we have multiplied the action by $\Delta t^{2}$ and ignored the constant $E\left(\boldsymbol{y}^{n}\right)$ in Eq. (11). This method is related to the backward-Euler method, and many other variational time-integrators can be defined by choosing different time-discrete approximations of the action. The resulting nonlinear optimization program can be solved with a variety of methods. Here, we impose the constraints with Lagrange multipliers and solve the first order optimality conditions with Newton's method, although an augmented Lagrangian method, combined with line-search may be more robust at very large time-steps. Note that for large time-steps, the objective function in Eq. (11) is dominated by the curvature energy, and the system nearly minimizes this energy in one step. On the contrary, small time-steps give more weight to the viscous dissipation, penalizing changes in the configuration of the system. Even though $E(\boldsymbol{y})$ has in general a complex, non-convex landscape, for a sufficiently small time-step, the viscous dissipation makes the objective function convex, and hence the nonlinear optimization problem becomes easier 
to solve. We also note that, by construction, $E\left(\boldsymbol{y}^{n+1}\right) \leq E\left(\boldsymbol{y}^{n}\right)$, and therefore the method is endowed automatically with nonlinear stability. Of course, the issue may be being able to numerically solve the nonlinear optimization problem.

We make the algorithm explicit in the dissipation matrix $\boldsymbol{K}(\boldsymbol{y})$, as otherwise the method is significantly more complex and most of the nonlinearity is in $E(\boldsymbol{y})$. In practical applications, we often update the Hessian of $E(\boldsymbol{y})$ once every time-increment, instead of in each iteration of Newton's method. We find that this significantly reduces the computational cost without affecting much the convergence of Newton's method. The treatment of the surface area constraint may be simplified by discretizing in time the linearized constraint, $\left[\nabla A^{h}\left(\boldsymbol{y}^{n}\right)\right]^{T}\left(\boldsymbol{y}-\boldsymbol{y}^{n}\right)=0$. However, this option leads to significant drifts in the surface area for large time-steps. Finally, we note that adaptive time-stepping algorithms can be easily designed, for instance adapting $\Delta t$ in such a way that $\Delta E$ is nearly constant. The adaptivity may also be driven by the number of iterations needed in the nonlinear solver.
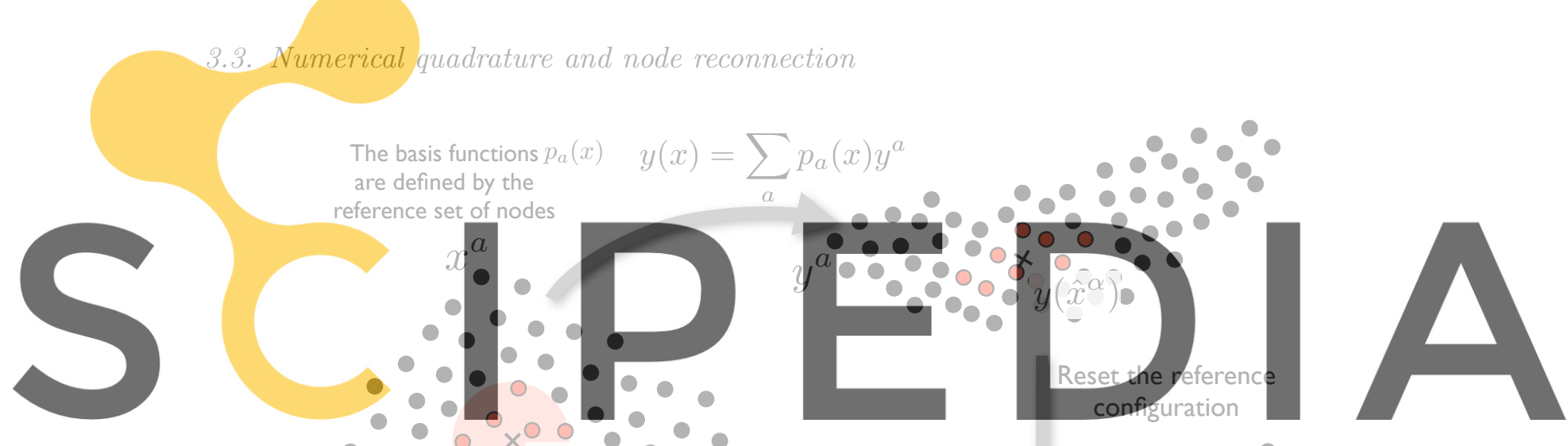

Register for free at https/Lwww.scipedir.com to download the version quithout the watermark

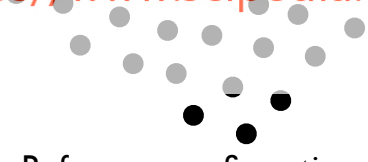

Reference configuration

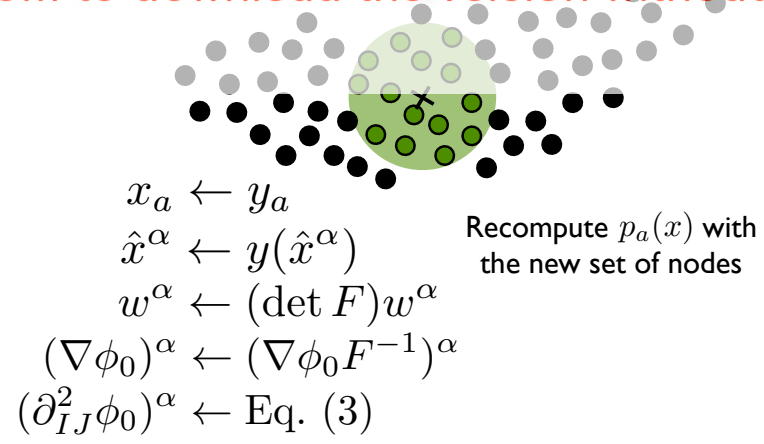

Figure 2: As the Lagrangian simulation proceeds, the deformation may significantly distort the domain. To avoid this, we periodically reset the reference configuration, as shown in the figure. This involves reseting the reference node position to the current position, recomputing the meshfree basis functions from the new node set, which involves new neighbor searches as indicated with the colored regions, and reseting the quadrature points $\hat{x}^{\alpha}$, the corresponding weights, and the reference phase-field first and second derivatives as indicated in the figure. Note that the reference phase-field value at the quadrature points, $\phi_{0}^{\alpha}$, does not need to be updated as the phase-field is a material property and the quadrature points keep their material identity. 
As discussed in Fig. 2, the evolution most likely produces large distortions, which may even lead to non-injective deformation mappings in Eq. (10), i.e. negative Jacobian determinants $\operatorname{det} F$. Even if the deformation map remains injective, it is a good idea to avoid excessive distortions, which degrade the accuracy of the approximation. For this reason, we periodically reset the reference configuration, reconnect the nodes, and build new basis functions to parameterize the deformation maps from the new reference configuration. The reconnection can be done when a measure of the distortion (a norm of $F$ ) exceeds a threshold, or simply every a fixed number of time steps. Numerical experience shows that frequent reconnection leads to better numerical accuracy, but also that the method is very robust and can deliver acceptable results with very few reconnections. In practice, the frequency of reconnection can be set by weighing accuracy and efficiency, although objective criteria would be desirable.

Note carefully that the reconnection procedure does not require any projection of fields if the multiplicative structure of the composition of maps is exploited. Indeed, suppose that the deformation map in the motion at which we decide to reset the reference configuration is $y(x)=\sum_{a} p_{a}(x) y^{a}$, and its deformation gradient $F$. The new node set is simply $\left\{y^{1}, \ldots, y^{N}\right\}$ and the new quadrature points are $y\left(\hat{x}^{\alpha}\right), \quad \alpha=1, \ldots, Q$. The value of the phase-field at these material points is simply the original value $\phi_{0}\left(\hat{x}^{\alpha}\right)$ since the phase-field is viewed as a material property. Its derivatives need to be updated with the formulas seen previously, but no new interpolation of the phase-field is needed and no new quadrature needs to be defined. The reset algorithm is sketched in Fig. 2. Remarkably, such reset of the reference configuration exactly preserves the elastic energy, area, and enclosed volume of the system, as can be understood from examining Eqs. $(5,6,7)$.

\section{Numerical examples}

We present next a set of numerical simulations to test the proposed method. We first illustrate the general performance of the method with regards to space and time adaptivity. The former is automatic if the initial grid adapts to the interface, while for the second we set the time-step such that the energy decrement per step is roughly constant. We compare the proposed variational time integration with explicit Euler time-stepping. We then describe three representative simulations of relaxation dynamics of vesicles initially placed out-of-equilibrium, showing large shape changes, and requiring multiple node reconnections. Finally, we evaluate kinetic effects on the shape trajectory by deflating a vesicle at different rates. In all examples, we consider 6 integration points per cell (the triangles of the Delaunay triangulation of the the initial set of nodes) and an aspect ratio parameter for the maximum-entropy basis functions of $\gamma=0.8$ [9]. The regularization parameter is chosen as about $1 \%$ of the size of the vesicle, i.e. $\epsilon / \sqrt{A_{0} /(4 \pi)}=0.01$.

Figure 3 shows (with a movie/with a collection of snapshots) the relaxation dynamics of an oblate vesicle brought out-of-equilibrium. The reduced volume, a non dimensional measure of the volume to area ratio, is $v=0.9$. We show the location of the nodes $y^{a}(t)$, and color-code them by the value of the phase-field. In this example, exhibiting moderate deformations, we do not reconnect the nodes and therefore the method is purely Lagrangian, with the initial configurations as a reference configuration during the whole motion. The calculation proceeds robustly despite the large deformations. It can be appreciated how the phase-field elastic energy 
maintains the transversal density of the nodes, and how the adapted region follows the features of the phase-field. We check the accuracy of this simulation with additional runs with a larger number of integration points, more nodes, and different $\gamma$ parameters.

The performance of the method is analyzed in Fig. 4. The left plot shows the non-dimensional energy $E^{*}=E / k$ and the non-dimensional time-step as a function of non-dimensional time $t^{*}=t k /\left(\mu R_{0}^{3}\right)$. The energy monotonically decreases as expected, converging towards the equilibrium energy calculated independently with a parametric method. As the process advances, the adaptive time-step grows to roughly keep the energy decrement per time-step constant. Remarkably, the time-step changes by two orders of magnitude during the simulation. At the final stages, the time-step hits the maximum allowed size.

Table 1: Elastic energy and computational cost for different constant time-steps and methods (VTI: variational time-integration, FE: forward Euler). $t_{1}^{*}=1.0 \cdot 10^{-3}, t_{2}^{*}=1.1 \cdot 10^{-2}$.

\begin{tabular}{lllllll}
\hline Method & $\Delta t^{*}$ & $E^{*}\left(t_{1}^{*}\right)$ & $E^{*}\left(t_{2}^{*}\right)$ & steps & grad & hess \\
\hline VTI & $1.0 \cdot 10^{-2}$ & 9.374 & 9.243 & 1 & 2 & 2 \\
VTI & $1.0 \cdot 10^{-3}$ & 9.374 & 9.240 & 10 & 20 & 10 \\
VTI & $1.0 \cdot 10^{-4}$ & 9.374 & 9.239 & 100 & 200 & 100 \\
FE & $1.0 \cdot 10^{-5}$ & 9.374 & 9.239 & 1000 & 1000 & 0 \\
\hline
\end{tabular}

Although more sophisticated time-stepping schemes are possible, as compare the proposed variational time-integration (VTI) method with an explicit forward Euler (FE) method. It is computationally infeasible to perform the full relaxation dynamics with the forward Euler method, which imposes very stringent conditions on the time-step. Instead, we focus on a portion of the dynamics, and report the results in Table 1. In the VTI method, we use Newton's method to numerically solve the optimization problem in Eq. (11), and for computational efficiency, update the Hessian matrix only once per time-step, not per iteration. However, for the largest time-step, we need to update the Hessian in each iteration for convergence. In all cases, Newton's method converges in two iterations. The table compares the VTI method with time-steps $\Delta t^{*}=$ $10^{-2}, 10^{-3}, 10^{-4}$, and the FE method with the largest time-step for stability in this interval, $\Delta t^{*}=10^{-5}$. The accuracy is reported in terms of the energy at the end of the interval, and the computational cost in terms of gradient and Hessian evaluations. The table shows the ability of VTI to robustly take large time-steps with accurate results. In contrast, we find that for this nonlinear system, it is very difficult to stably adjust the time-step length in the FE method. We find that the VTI method provides a similar accuracy to the explicit method with time-steps between one and two orders of magnitude larger. This ratio is even more dramatic in the initial fast stages of the dynamics.

We next exercise the method in more challenging dynamics, involving large shape changes. Figure 5 (left) shows a stomatocyte-discocyte dynamical transition. For the considered reduced volume $v=0.6$, both a stomatocyte and a discocyte are metastable configurations, the latter having lower energy. We slightly displace the stomatocyte equilibrium configuration beyond the energy barrier, and then the system spontaneously evolves towards the discocyte configuration. The reference configuration is reset, as illustrated in Fig. 2, when large distortions occur as measured with the gradient of deformation mapping. In this simulation, the reference configuration 
is reset every 20 time-steps. The time-adaptive scheme allows us to efficiently track the entire transition, and by the end of the simulation the time-step is 2,048 times the initial time-step. Figure 5 (right) shows the response of an prolate vesicle $(v=0.8)$ subject to an instantaneous change of spontaneous curvature from $C_{0}=0$ to $C_{0} \sqrt{A_{0} /(4 \pi)}=10.0$, which can be the result of exposing the bilayer to a different chemical environment $[5,6,27]$. The system evolves towards a configuration consisting of two dissimilar spheres connected by a narrow neck, which best adjusts to the imposed spontaneous curvature with the available volume. Both simulations run on a CVT adapted grid of 6,124 nodes.

Figure 6 shows an even more dramatic shape change, in which a prolate vesicle is deflated from $v=0.9$ to $v=0.55$ and its spontaneous curvature increased to $C_{0} \sqrt{A_{0} /(4 \pi)}=12.0$, leading to an elongation and pearling transformation, widely observed in experiments [41]. The method robustly follows all the large shape deformations with an adapted grid of 12,650 nodes. This simulation requires frequent nodal reconnection, and even four complete re-meshing steps at later stages, in which a new grid is built and adapted to the current phase-field and the phase-field is projected onto the new grid.

Finally, we present a series of simulations highlighting kinetic effects. By subjecting a vesicle to fast changes (here a volume decrease rate), the system follows an out-of-equilibrium path that significantly deviates from the quasi-static response. We then fix the enclosed volume, and let the system relax towards equilibrium. In Fig. 7, we report the response of the system to three different volume decrease rates in terms of elastic energy evolution and shape at the instant of maximum energy for each evolution, which corresponds to the end of the deflection process. It can be observed that, due to the fluid dissipative forces, the faster the dynamics, the further apart is the shape at this instant from the equilibrium shape (D), eventually reached by all the simulations for $T^{*} \approx 1.00$. Also, the faster the rate, the larger the deviation between the elastic energy at this instant and the elastic energy in equilibrium. In principle, kinetic effects such as those reported here could assist in the transition to a different equilibrium branch, and bring the system to a qualitatively different equilibrium configuration. We are currently exploring such phenomena.

\section{Conclusions}

We have proposed an adaptive meshfree Galerkin method to numerically approximate the dynamics phase-field models of biomembranes embedded in a viscous fluid. We have shown the ability of the proposed method, based on smooth approximants, to deal with the high order character of the equations in a direct manner. Furthermore, adaptivity is very natural for a meshfree method, and proves essential to resolve the sharp features of the phase-field model at an affordable cost. We have presented an original Lagrangian and variational formulation of the coupled fluid-membrane dynamics, which lends itself to efficient and robust time integrators based on time-incremental minimization problems. In this method, the local refinement follows naturally the sharp features of the phase-field. This combination of methods shows promise of robust, scalable computations of complex membrane systems in three dimensions, currently under development. 


\section{Acknowledgments}

We acknowledge the support of the European Research Council under the European Community's 7th Framework Programme (FP7/2007-2013)/ERC grant agreement nr 240487, and of the Ministerio de Ciencia e Innovacin (DPI2011-26589). MA acknowledges the support received through the prize "ICREA Academia" for excellence in research, funded by the Generalitat de Catalunya. CP acknowledges FPI-UPC Grant, FPU Ph. D. Grant (Ministry of Science and. Innovation, Spain) and Col-legi d'Enginyers de Camins, Canals i Ports de Catalunya for their support.

\section{Appendix A. Cylindrical coordinates}

We consider cylindrical coordinates, but assume that there is no angular dependence of any function along the angular direction. We have $x=(R, Z, \Theta)$, and $y(x)=(r(R, Z), z(R, Z), \Theta)$. The metric tensors of the reference and the deformed coordinate systems are

$$
G_{I J}=\left(\begin{array}{ccc}
1 & 0 & 0 \\
0 & 1 & 0 \\
0 & 0 & R^{2}
\end{array}\right), \quad g_{i j}=\left(\begin{array}{ccc}
1 & 0 & 0 \\
0 & 1 & 0 \\
0 & 0 & r^{2}
\end{array}\right) .
$$

It follows immediately that the volume element can be written as $d v=r d r d z d \theta, d V=R d R d Z d \Theta$. The deformation gradient becomes

$$
F_{I}^{i}=\left(\begin{array}{ccc}
r_{, R} & r_{, Z} & 0 \\
z_{, R} & z_{, Z} & 0 \\
0 & 0 & 1
\end{array}\right)
$$

where the comma denotes partial differentiation. We denote by $\tilde{F}$ its first $2 \times 2$ minor. The Cauchy-Green deformation tensor can be written as $C^{I}{ }_{J}=F^{i}{ }_{K} F^{j}{ }_{J} g_{i j} G^{I K}$ [35]. Consequently, exploiting the block structure,

$$
J=\sqrt{\operatorname{det} C_{J}^{I}}=(r / R) \operatorname{det} \tilde{F}
$$

Thus, we have

$$
d v=J d V=r(\operatorname{det} \tilde{F}) d R d Z d \Theta .
$$

For a function $\phi_{0}(R, Z)$, the gradient and the differential components coincide

$$
\phi_{0, J} G^{I J}=\phi_{0, I}=\left(\begin{array}{c}
\phi_{0, R} \\
\phi_{0, Z} \\
0
\end{array}\right) .
$$

By the chain rule, recalling that $\phi=\phi_{0} \circ y^{-1}$, we have

$$
\phi_{, i}=\left(F^{-1}\right)^{I}{ }_{i} \phi_{0, I},
$$


which reduces in the $(R, Z)$ plane to

$$
\tilde{\phi}_{, i}=\left(\tilde{F}^{-1}\right)^{I}{ }_{i} \tilde{\phi}_{0, I},
$$

owing to the block structure of the formation gradient and the fact that $\phi_{0, \theta}=0$.

To compute covariant derivatives of vector fields and one-forms

$$
v^{i}{ }_{\mid j}=v_{, j}^{i}+\gamma_{j k}^{i} v^{k}, \quad \alpha_{i \mid j}=\alpha_{i, j}-\gamma_{i j}^{k} \alpha_{k},
$$

we need the connection coefficients, which can be computed from

$$
\gamma_{j k}^{i}=(1 / 2) g^{i l}\left(g_{l j, k}+g_{l k, j}-g_{j k, l}\right) .
$$

The only non-zero components are

$$
\gamma_{\theta \theta}^{r}=-r, \quad \gamma_{r \theta}^{\theta}=\gamma_{\theta r}^{\theta}=1 / r .
$$

For a vector field $v(r, z)=\left(v_{r}(r, z), v_{z}(r, z), 0\right)$, we can compute the covariant derivative

$$
(\nabla v)^{i}{ }_{j}=v^{i}{ }_{\mid j}=\left(\begin{array}{ccc}
v^{r}{ }_{, r} & v^{r}{ }_{, z} & 0 \\
v^{z},{ }_{, r} & v^{z}{ }_{, z} & 0 \\
0 & 0 & v^{r} / r
\end{array}\right),
$$

and taking the trace, its divergence

$$
\operatorname{div} v=v^{r}{ }_{, r}+v^{z}, z+v^{r} / r \text {. }
$$

Now, from the above expressions, we can compute the Hessian of $\phi$ as

$$
\phi_{\mid i j}=\phi_{, i j}-\gamma_{i j}^{l} \phi_{, l} .
$$

Again, it has a block diagonal structure

$$
\phi_{\mid i j}=\left(\begin{array}{c|c}
\tilde{\phi}_{\mid i j} & 0 \\
\hline 0 & \phi_{\mid \theta \theta}
\end{array}\right) .
$$

In the first $2 \times 2$ minor, the Cartesian structure given in Eq. (3) is preserved, while in the $\theta \theta$ component we have

$$
\phi_{\mid \theta \theta}=r \phi_{, r}=r\left[\left(F^{-1}\right)_{r}^{R} \phi_{0, R}+\left(F^{-1}\right)_{r}^{Z} \phi_{0, Z}\right] .
$$

The Laplacian is computed correspondingly as

$$
\Delta \phi=\phi_{\mid i j} g^{i j}=\Delta \tilde{\phi}+\phi_{, r} / r=\Delta \tilde{\phi}+(1 / r)\left[\left(F^{-1}\right)^{R}{ }_{r} \phi_{0, R}+\left(F^{-1}\right)_{r}^{Z} \phi_{0, Z}\right] .
$$

The Lagrangian expression of the rate-of-deformation tensor can be computed as [35]

$$
2 D_{I J}=g_{i k}\left(V_{\mid I}^{k} F_{J}^{i}+V_{\mid J}^{i} F_{I}^{k}\right),
$$


where the covariant derivative of the material velocity is defined as

$$
V_{\mid J}^{i}=V_{, J}^{i}+\gamma_{j k}^{i} V^{j} F^{k} .
$$

This tensor is simply

$$
V_{\mid J}^{i}=\left(\begin{array}{ccc}
V^{r}, R & V^{r}, Z & 0 \\
V^{z}, R & V^{z}, Z & 0 \\
0 & 0 & V^{r} / r
\end{array}\right)
$$

which leads to

$$
2 D_{I J}=\left(\begin{array}{c|c}
\tilde{V}_{, I}^{k} \tilde{F}_{, J}^{k}+\tilde{V}_{, J}^{k} \tilde{F}_{, I}^{k} & 0 \\
\hline 0 & 2 r V^{r}
\end{array}\right)=\left(\begin{array}{c|c}
\dot{\tilde{F}}^{T} \tilde{F}+\tilde{F}^{T} \dot{\tilde{F}} & 0 \\
\hline 0 & 2 r V^{r}
\end{array}\right) .
$$

Now, noting that the Eulerian rate-of-deformation tensor can be computed as $d_{i j} \circ y=D_{I J}\left(F^{-1}\right)^{I}{ }_{i}\left(F^{-1}\right)^{J}{ }_{j}$ [35], we have

$$
2 d_{i j} \circ y=\left(\begin{array}{c|c}
\tilde{F}^{-T} \dot{\tilde{F}}^{T}+\dot{\tilde{F}} \tilde{F}^{-1} & 0 \\
\hline 0 & 2 r \dot{r}
\end{array}\right) .
$$

Its trace can be computed as

$$
(\operatorname{div} v) \circ y=\left(d_{i j} g^{i j}\right) \circ y=(1 / 2) \operatorname{trace}\left(\tilde{F}^{-T} \dot{\tilde{F}}^{T}+\dot{\tilde{F}} \tilde{F}^{-1}\right)+\dot{r} / r,
$$

while its norm squared is

$$
|d \circ y|^{2}=\left(d_{i j} d_{k l} g^{i k} g^{j l}\right) \circ y=(1 / 4)\left|\tilde{F}^{-T} \dot{\tilde{F}}^{T}+\dot{\tilde{F}} \tilde{F}^{-1}\right|^{2}+(\dot{r} / r)^{2} .
$$

\section{Appendix B. Derivatives for gradient and Hessian of the energy, volume, and area}

The main expressions and derivatives required to implement the proposed algorithm are presented in this appendix. We start with the derivatives of the motion, then move to nodal derivatives involving the gradient, and finish with the Hessian, used in Newton's method. Lighter gray symbols correspond to terms required in the axisymmetric formulation, which just need to be dropped in $3 \mathrm{D}$.

\section{Appendix B.1. Spacial derivatives of the motion}

The motion is discretized as

$$
y(x, t)=\sum_{a=1}^{N} p^{a}(x) y^{a}(t)
$$

From now on, we ignore the arguments of the basis functions and nodal values for simplicity, $y=\sum_{a=1}^{N} p^{a} y^{a}$. We then have for the deformation gradient

$$
F_{i I}=\sum_{a=1}^{N} \partial_{I} p^{a} y_{i}^{a}, \quad \partial_{J} F_{i I}=\sum_{a=1}^{N} \partial_{I} \partial_{J} p^{a} y_{i}^{a}, \quad \partial_{J} F_{I i}^{-1}=-F_{I l}^{-1} F_{K i}^{-1} \partial_{J} F_{l K} .
$$


Appendix B.2. Nodal derivatives of the motion

Nodal derivative of $F$ and $F^{-1}$

$$
\partial_{y_{k}^{b}} F_{i I}=\sum_{a=1}^{N} \partial_{I} p^{a} \partial_{y_{k}^{b}} y_{i}^{a}=\partial_{I} p^{b} \partial_{y_{k}^{b}} y_{i}^{b}=\partial_{I} p^{b} \delta_{i k}, \quad \partial_{y_{k}^{b}} F_{I i}^{-1}=-F_{I l}^{-1} \partial_{y_{k}^{b}} F_{l K} F_{K i}^{-1} .
$$

Nodal derivative of $\nabla F$

$$
\partial_{y_{k}^{b}} \partial_{J} F_{i I}=\sum_{a=1}^{N} \partial_{I} \partial_{J} p^{a} \partial_{y_{k}^{b}} y_{i}^{a}=\partial_{I} \partial_{J} p^{b} \partial_{y_{k}^{b}} y_{i}^{b}=\partial_{I} \partial_{J} p^{b} \delta_{i k}
$$

Nodal derivative of $\nabla F^{-1}$

$$
\partial_{y_{k}^{b}} \partial_{J} F_{i I}^{-1}=-\left(\partial_{y_{k}^{b}} F_{I l}^{-1} F_{K i}^{-1} \partial_{J} F_{i I}+F_{I l}^{-1} F_{K i}^{-1} \partial_{y_{k}^{b}} \partial_{J} F_{i I}+F_{I l}^{-1} \partial_{y_{k}^{b}} F_{K i}^{-1} \partial_{J} F_{i I}\right) .
$$

Nodal derivative of $\operatorname{det} F$

$$
\partial_{y_{k}^{b}} \operatorname{det} F=\operatorname{det} F\left(F_{I i}^{-1} \partial_{y_{k}^{b}} F_{i I}\right)
$$

Second nodal derivative of $F^{-1}$

$$
\partial_{y_{j}^{a}} \partial_{y_{k}^{b}} F_{I i}^{-1}=-\left(\partial_{y_{j}^{a}} F_{I l}^{-1} \partial_{y_{k}^{b}} F_{l K} F_{K i}^{-1}+F_{I l}^{-1} \partial_{y_{k}^{b}} F_{l K} \partial_{y_{j}^{a}} F_{K i}^{-1}\right)
$$

Second nodal derivative of $\nabla F^{-1}$

$$
\begin{array}{r}
\partial_{y_{j}^{a}} \partial_{y_{k}^{b}} \partial_{J} F_{I i}^{-1}=-\left(\partial_{y_{j}^{a}} \partial_{y_{k}^{b}} F_{I l}^{-1} F_{K i}^{-1} \partial_{J} F_{l K}+\partial_{y_{k}^{b}} F_{I l}^{-1} \partial_{y_{j}^{a}} F_{K i}^{-1} \partial_{J} F_{l K}+\partial_{y_{k}^{b}} F_{I l}^{-1} F_{K i}^{-1} \partial_{y_{j}^{a}} \partial_{J} F_{l K}+\right. \\
\partial_{y_{j}^{a}} F_{I l}^{-1} F_{K i}^{-1} \partial_{y_{k}^{b}} \partial_{J} F_{l K}+F_{I l}^{-1} \partial_{y_{j}^{a}} F_{K i}^{-1} \partial_{y_{k}^{b}} \partial_{J} F_{l K}+ \\
\left.\partial_{y_{j}^{a}} F_{I l}^{-1} \partial_{y_{k}^{b}} F_{K i}^{-1} \partial_{J} F_{l K}+F_{I l}^{-1} \partial_{y_{j}^{a}} \partial_{y_{k}^{b}} F_{K i}^{-1} \partial_{J} F_{l K}+F_{I l}^{-1} \partial_{y_{k}^{b}} F_{K i}^{-1} \partial_{y_{j}^{a}} \partial_{J} F_{l K}\right)
\end{array}
$$

Second nodal derivative of $\operatorname{det} F$

$$
\partial_{y_{j}^{a}} \partial_{y_{k}^{b}} \operatorname{det} F=\operatorname{det} F\left(F_{I i}^{-1} \partial_{y_{k}^{b}} F_{i I}\right)\left(F_{M m}^{-1} \partial_{y_{j}^{a}} F_{m M}\right)-\operatorname{det} F\left(F_{I m}^{-1} \partial_{y_{j}^{a}} F_{m K} F_{K i}^{-1} \partial_{y_{k}^{b}} F_{i I}\right)
$$

Appendix B.3. Derivatives of the phase-field

From the numerical discretization of the reference phase-field

$$
\phi_{0}(x)=\sum_{a=1}^{N} p^{a}(x) \phi_{0}^{a}
$$

we have

$$
\partial_{I} \phi_{0}=\sum_{a=1}^{N} \partial_{I} p^{a} \phi_{0}^{a}, \quad \partial_{I} \partial_{J} \phi_{0}=\sum_{a=1}^{N} \partial_{I} \partial_{J} p^{a} \phi_{0}^{a}
$$

Consequently, we can compute the gradient and Hessian $(2 \times 2$ minor for axisymmetry or full tensor in 3D) of the deformed phase-field

$$
\partial_{i} \phi=\partial_{I} \phi_{0} F_{I i}^{-1}, \quad \partial_{i} \partial_{j} \phi=\partial_{I} \partial_{J} \phi_{0} F_{I i}^{-1} F_{J j}^{-1}+\partial_{I} \phi_{0} \partial_{J} F_{I i}^{-1} F_{J j}^{-1}
$$

Its Laplacian becomes

$$
\Delta \phi=\partial_{I} \partial_{J} \phi_{0} F_{I i}^{-1} F_{J i}^{-1}+\partial_{I} \phi_{0} \partial_{J} F_{I i}^{-1} F_{J i}^{-1}+\phi_{, r} / r
$$


Appendix B.4. Nodal derivatives of the energy, volume and area

Defining for convenience

$$
C_{1}=\left(\phi_{0} / \epsilon+c_{0} \sqrt{2}\right)\left(1-\phi_{0}^{2}\right), \quad C_{2}=\frac{1}{4 \epsilon}\left(1-\phi_{0}^{2}\right)^{2}, \quad W=\epsilon \Delta \phi+C_{1},
$$

we have

$$
\begin{gathered}
E=f_{E} \int_{\Omega_{0}} W^{2} \operatorname{det} F r d \Omega_{0}, \\
A=f_{A} \int_{\Omega_{0}}\left[\frac{\epsilon}{2}|\nabla \phi|^{2}+C_{2}\right] \operatorname{det} F r d \Omega_{0}, \\
V=\frac{1}{2}\left[\operatorname{Vol}\left(\Omega_{0}\right)+\int_{\Omega_{0}} \phi_{0} \operatorname{det} F r d \Omega_{0}\right],
\end{gathered}
$$

where $f_{E}=3 k /(16 \sqrt{2} \epsilon)$ and $f_{A}=3 /(2 \sqrt{2})$.

The gradient of the energy then follows as

$$
\partial_{y_{j}^{b}} E=f_{E} \int_{\Omega_{0}}\left(2 W \partial_{y_{j}^{b}} W \operatorname{det} F+W^{2} \partial_{y_{j}^{b}} \operatorname{det} F\right) r+W^{2} \operatorname{det} F \partial_{y_{j}^{b}} r d \Omega_{0}
$$

where

$$
\begin{aligned}
\partial_{y_{j}^{b}} W / \epsilon= & \partial_{y_{j}^{b}} \partial_{i} \partial_{i} \phi=\partial_{I} \partial_{J} \phi_{0} \partial_{y_{j}^{b}} F_{I i}^{-1} F_{J i}^{-1}+\partial_{I} \partial_{J} \phi_{0} F_{I i}^{-1} \partial_{y_{j}^{b}} F_{J i}^{-1}+ \\
& \partial_{I} \phi_{0} \partial_{y_{j}^{b}} \partial_{J} F_{I i}^{-1} F_{J i}^{-1}+\partial_{I} \phi_{0} \partial_{J} F_{I i}^{-1} \partial_{y_{j}^{b}} F_{J i}^{-1}+\partial_{y_{j}^{b}} \phi_{, r} / r-\left(\phi_{, r} / r^{2}\right) \partial_{y_{j}^{b}} r
\end{aligned}
$$

The gradient of the area is

$$
\begin{aligned}
\partial_{y_{j}^{b}} A= & f_{A} \int_{\Omega_{0}}\left[\frac{\epsilon}{2} \partial_{y_{j}^{b}}|\nabla \phi|^{2} \operatorname{det} F+\left(\frac{\epsilon}{2}|\nabla \phi|^{2}+C_{2}\right) \partial_{y_{j}^{b}} \operatorname{det} F\right] r \\
& +\left(\frac{\epsilon}{2}|\nabla \phi|^{2}+C_{2}\right) \operatorname{det} F \partial_{y_{j}^{b}} r d \Omega_{0},
\end{aligned}
$$

where,

$$
\begin{aligned}
\partial_{y_{j}^{b}}|\nabla \phi|^{2} & =\partial_{y_{j}^{b}} \partial_{i} \phi \partial_{i} \phi=\partial_{I} \phi_{0} \partial_{y_{j}^{b}} F_{I i}^{-1} \partial_{J} \phi_{0} F_{J i}^{-1}+\partial_{I} \phi_{0} F_{I i}^{-1} \partial_{J} \phi_{0} \partial_{y_{j}^{b}} F_{J i}^{-1} \\
& =2 \partial_{I} \phi_{0} F_{I i}^{-1} \partial_{J} \phi_{0} \partial_{y_{j}^{b}} F_{J i}^{-1} .
\end{aligned}
$$

Finally, the derivative of the volume is

$$
\partial_{y_{j}^{b}} V=\frac{1}{2} \int_{\Omega_{0}}\left(\phi_{0} \partial_{y_{j}^{b}} \operatorname{det} F\right) r+\phi_{0} \operatorname{det} F \partial_{y_{j}^{b}} r d \Omega_{0} .
$$

We can compute the Hessian of the energy as

$$
\begin{aligned}
\partial_{y_{i}^{a}} \partial_{y_{j}^{b}} E= & f_{E} \int_{\Omega_{0}}\left(2 W \partial_{y_{i}^{a}} \partial_{y_{j}^{b}} W \operatorname{det} F+2 \partial_{y_{i}^{a}} W \partial_{y_{j}^{b}} W \operatorname{det} F\right. \\
& \left.+2 W \partial_{y_{j}^{b}} W \partial_{y_{i}^{a}} \operatorname{det} F+2 W \partial_{y_{i}^{a}} W \partial_{y_{j}^{b}} \operatorname{det} F+W^{2} \partial_{y_{i}^{a}} \partial_{y_{j}^{b}} \operatorname{det} F\right) r \\
& +\left(2 W \partial_{y_{j}^{b}} W \operatorname{det} F+W^{2} \partial_{y_{j}^{b}} \operatorname{det} F\right) \partial_{y_{i}^{a}} r+\left(2 W \partial_{y_{i}^{a}} W \operatorname{det} F+W^{2} \partial_{y_{i}^{a}} \operatorname{det} F\right) \partial_{y_{j}^{b}} r d \Omega_{0},
\end{aligned}
$$


where,

$$
\begin{aligned}
\partial_{y_{i}^{a}} \partial_{y_{j}^{b}} W / \epsilon= & \partial_{I} \partial_{J} \phi_{0} \partial_{y_{i}^{a}} \partial_{y_{j}^{b}} F_{I k}^{-1} F_{J k}^{-1}+\partial_{I} \partial_{J} \phi_{0} \partial_{y_{j}^{b}} F_{I k}^{-1} \partial_{y_{i}^{a}} F_{J k}^{-1}+\partial_{I} \partial_{J} \phi_{0} \partial_{y_{i}^{a}} F_{I k}^{-1} \partial_{y_{j}^{b}} F_{J k}^{-1} \\
& +\partial_{I} \partial_{J} \phi_{0} F_{I k}^{-1} \partial_{y_{i}^{a}} \partial_{y_{j}^{b}} F_{J k}^{-1}+\partial_{I} \phi_{0} \partial_{y_{i}^{a}} \partial_{y_{j}^{b}} \partial_{J} F_{I k}^{-1} F_{J k}^{-1}+\partial_{I} \phi_{0} \partial_{y_{j}^{b}} \partial_{J} F_{I k}^{-1} \partial_{y_{i}^{a}} F_{J k}^{-1} \\
& +\partial_{I} \phi_{0} \partial_{y_{i}^{a}} \partial_{J} F_{I k}^{-1} \partial_{y_{j}^{b}} F_{J k}^{-1}+\partial_{I} \phi_{0} \partial_{J} F_{I k}^{-1} \partial_{y_{i}^{a}} \partial_{y_{j}^{b}} F_{J k}^{-1} \\
& +\partial_{y_{i}^{a}} \partial_{y_{j}^{b}} \phi_{, r} / r-\left(1 / r^{2}\right) \partial_{y_{i}^{a}} \phi_{, r} \partial_{y_{j}^{b}} r-\left(1 / r^{2}\right) \partial_{y_{j}^{b}} r \partial_{y_{i}^{a}} \phi_{, r}+\left(2 \phi_{, r} / r^{3}\right) \partial_{y_{i}^{a}} r \partial_{y_{j}^{b}} r .
\end{aligned}
$$

For the area, we have

$$
\begin{aligned}
\partial_{y_{i}^{a}} \partial_{y_{j}^{b}} A= & f_{A} \int_{\Omega_{0}}\left[\frac{\epsilon}{2} \partial_{y_{i}^{a}} \partial_{y_{j}^{b}}|\nabla \phi|^{2} \operatorname{det} F+\left(\frac{\epsilon}{2}|\nabla \phi|^{2}+C_{2}\right) \partial_{y_{i}^{a}} \partial_{y_{j}^{b}} \operatorname{det} F\right. \\
& \left.+\frac{\epsilon}{2} \partial_{y_{j}^{b}}|\nabla \phi|^{2} \partial_{y_{i}^{a}} \operatorname{det} F+\frac{\epsilon}{2} \partial_{y_{i}^{a}}|\nabla \phi|^{2} \partial_{y_{j}^{b}} \operatorname{det} F\right] r \\
& +\left[\frac{\epsilon}{2} \partial_{y_{i}^{a}}|\nabla \phi|^{2} \operatorname{det} F+\left(\frac{\epsilon}{2}|\nabla \phi|^{2}+C_{2}\right) \partial_{y_{i}^{a}} \operatorname{det} F\right] \partial_{y_{j}^{b} r} \\
& +\left[\frac{\epsilon}{2} \partial_{y_{j}^{b}}|\nabla \phi|^{2} \operatorname{det} F+\left(\frac{\epsilon}{2}|\nabla \phi|^{2}+C_{2}\right) \partial_{y_{j}^{b}} \operatorname{det} F\right] \partial_{y_{i}^{a}} r d \Omega_{0}
\end{aligned}
$$

where,

$$
\partial_{y_{i}^{a}} \partial_{y_{j}^{b}}|\nabla \phi|^{2}=2 \partial_{I} \phi_{0} \partial_{y_{i}^{a}} F_{I k}^{-1} \partial_{J} \phi_{0} \partial_{y_{j}^{b}} F_{J k}^{-1}+2 \partial_{I} \phi_{0} F_{I k}^{-1} \partial_{J} \phi_{0} \partial_{y_{i}^{a}} \partial_{y_{j}^{b}} F_{J k}^{-1}
$$

Finally,

$$
\partial_{y_{i}^{a}} \partial_{y_{j}^{b}} V=\frac{1}{2} \int_{\Omega_{0}} \phi_{0} \partial_{y_{i}^{a}} \partial_{y_{j}^{b}} \operatorname{det} F r+\phi_{0} \partial_{y_{i}^{a}} \operatorname{det} F \partial_{y_{j}^{b}} r+\phi_{0} \partial_{y_{j}^{b}} \operatorname{det} F \partial_{y_{i}^{a}} r d \Omega_{0}
$$

[1] M. K. W. Wintz, U. Seifert, R. Lipowsky, Fluid vesicles in shear flow, Physical Review Letters 77 (17) (1996) 3685-3688.

[2] U. Seifert, Fluid membranes in hdrodynamic flow fields: Formalism and an application to fluctuating quasispherical vesicles in shear flow., Eur. Phys. J. B 8 (1999) 405-415.

[3] H. Noguchi, G. Gompper, Dynamics of fluid vesicles in shear flow: Effect of membrane viscosity and thermal fluctuations, Physical Review E 72 (2005) 011901.

[4] S. Veerapaneni, A. Rahimian, G. Biros, D. Zorin, A fast algorithm for simulating vesicle flows in three dimensions, Journal of Computational Physics 230 (2011) 5610-5634.

[5] J. B. Fournier, N. Khalifat, N. Puff, M. I. Angelova, Chemically triggered ejection of membrane tubules controlled by intermonolayer friction, Phys. Rev. Lett. 102 (2009) 018102.

[6] N. Khalifat, N. Puff, S. Bonneau, J.-B. Fournier, M. I. Angelova, Membrane deformation under local ph gradient: Mimicking mitochondrial cristae dynamics, Biophys. J. 95 (10) (2008) 4924-4933.

[7] M. Staykova, M. Arroyo, M. Rahimi, S. H. A, Confined bilayers passively regulate shape and stress, Phys. Rev. Lett. 110 (2013) 028101. 
[8] M. Rahimi, M. Arroyo, Shape dynamics, lipid hydrodynamics, and the complex viscoelasticity of bilayer membranes, Physical Review E 86 (2012) 011932.

[9] A. Rosolen, C. Peco, M. Arroyo, An adaptive meshfree method for phase-field models of biomembranes. Part I: approximation with maximum-entropy approximants, Journal of Computational Physics ?? (2013) ??-??

[10] M. Arroyo, M. Ortiz, Local maximum-entropy approximation schemes: a seamless bridge between finite elements and meshfree methods, International Journal for Numerical Methods in Engineering 65 (13) (2006) 2167-2202.

[11] B. Li, F. Habbal, M. Ortiz, Optimal transportation meshfree approximation schemes for fluid and plastic flows, International Journal for Numerical Methods in Engineering 83 (12) (2010) 1541-1579.

[12] J. L. McWhirter, H. Noguchi, G. Gompper, Flow-induced clustering and alignment of vesicles and red blood cells in microcapillaries, Proceedings of the National Academy of Sciences 106 (15) (2009) 6039-6043.

[13] A. Bonito, R. Nochetto, S. Pauletti, Parametric FEM for geometric biomembranes, Journal of Computational Physics 229 (9) (2010) 3171-3188.

[14] A. Bonito, R. H. Nochetto, M. S. Pauletti, Dynamics of biomembranes: Effect of the bulk fluid, Mathematical Modelling of Natural Phenomena 6 (2011) 25-43.

[15] S. Veerapaneni, D. Gueyffier, G. Biros, D. Zorin, A numerical method for simulating the dynamics of $3 \mathrm{~d}$ axisymmetric vesicles suspended in viscous flows, Journal of Computational Physics 228 (19) (2009) 7233-7249.

[16] C. S. Peskin, The immersed boundary method, Acta Numer. 11 (2002) 479-517.

[17] D. V. Le, J. White, J. Peraire, K. M. Lim, B. C. Khoo, An implicit immersed boundary method for three-dimensional fluid-membrane interactions, Journal of Computational Physics 228 (2009) 8427-8445.

[18] Y. Kim, M.-C. Lai, Simulating the dynamics of inextensible vesicles by the penalty immersed boundary method, Journal of Computational Physics 229 (2010) 4840-4853.

[19] Q. Du, C. Liu, X. Wang, A phase field approach in the numerical study of the elastic bending energy for vesicle membranes, Journal of Computational Physics 198 (2004) 450-468.

[20] X. Wang, Phase field models and simulations of vesicle bio-membranes, Ph.D. thesis, Department of Mathematics, The Pennsylvania State University, Pennsylvania, USA (2005).

[21] Q. Du, C. Liu, R. Ryham, X. Wang, Energetic variational approaches in modeling vesicle and fluid interactions, Physica D 238 (2009) 923-930.

[22] T. Biben, K. Kassner, C. Misbah, Phase-field approach to three-dimensional vesicle dynamics, Physical Review E 72 (4) (2005) 041921. 
[23] D. Jamet, C. Misbah, Towards a thermodynamically consistent picture of the phase-field model of vesicles: Local membrane incompressibility, Phys. Rev. E 76 (2007) 051907.

[24] M. Farshbaf-Shaker, H. Garcke, Thermodynamically consistent higher order phase field Navier-Stokes models with applications to biomembranes., Discrete Contin. Dyn. Syst., Ser. S 4 (2011) 371-389.

[25] Q. Du, Phase field calculus, curvature-dependent energies, and vesicle membranes, Philosophical Magazine 91 (2010) 165-181.

[26] L. Ma, W. Klug, Viscous regularization and r-adaptive remeshing for finite element analysis of lipid membrane mechanics, Journal of Computational Physics 227 (11) (2008) 5816 5835 .

[27] F. Campelo, Modeling morphological instabilities in lipid membranes with anchored amphiphilic polymers, J. Chem. Biol. 2 (2009) 65-80.

[28] Q. Du, J. Zhang, Adaptive finite element method for a phase field bending elasticity model of vesicle membrane deformations, SIAM J. Sci. Comput. 30 (3) (2008) 1634-1657.

[29] A. Rosolen, D. Millán, M. Arroyo, Second order convex maximum entropy approximants with applications to high order PDE, International Journal for Numerical Methods in Engineering 94 (2) (2013) 150-182.

[30] E. Oñate, M. A. Celigueta, S. R. Idelsohn, F. Salazar, B. Suárez, Possibilities of the particle finite element method for fluid-soil-structure interaction problems, Computational Mechanics 48 (2011) 307-318.

[31] H. Goldstein, C. Poole, J. Safko, Classical Mechanics, Addison-Wesley, 2001.

[32] A. Lew, J. E. Marsden, M. Ortiz, M. West, Variational time integrators, Internat. J. Numer. Methods Engrg. 60 (2004) 153-212.

[33] M. Ortiz, E. A. Repetto, Nonconvex energy minimization and dislocation structures in ductile single crystals, Journal of the Mechanics and Physics of Solids 47 (1999) 397-462.

[34] Q. Du, C. Liu, X. Wang, Simulating the deformation of vesicle membranes under elastic bending energy in three dimensions, Journal of Computational Physics 212 (2006) 757-777.

[35] J. Marsden, T. Hughes, The mathematical foundations of elasticity, Prentice-Hall, 1983.

[36] T. Belytschko, W. Liu, B. Moran, Nonlinear Finite Elements for Continua and Structures, John Wiley \& Sons, England, 2001.

[37] C. Peco, A. Rosolen, M. Arroyo, Stabilized analysis of Stokes's equations with local maximum entropy meshfree approximantsIn preparation.

[38] J. Happel, H. Brenner, Low Reynolds Number Hydrodynamics: with special applications to particulate media, Martinus Nijhoff Publishers, 1983. 
[39] M. Arroyo, A. DeSimone, Relaxation dynamics of fluid membranes, Phys. Rev. E 79 (3) (2009) 031915.

[40] A. Rosolen, D. Millán, M. Arroyo, On the optimum support size in meshfree methods: a variational adaptivity approach with maximum entropy approximants, International Journal for Numerical Methods in Engineering 82 (7) (2010) 868-895.

[41] J. Sanborn, K. Oglecka, R. S. Kraut, A. N. Parikh, Transient pearling and vesiculation of membrane tubes under osmotic gradients, Faraday Discussions DOI: 10.1039/C2FD20116J. 

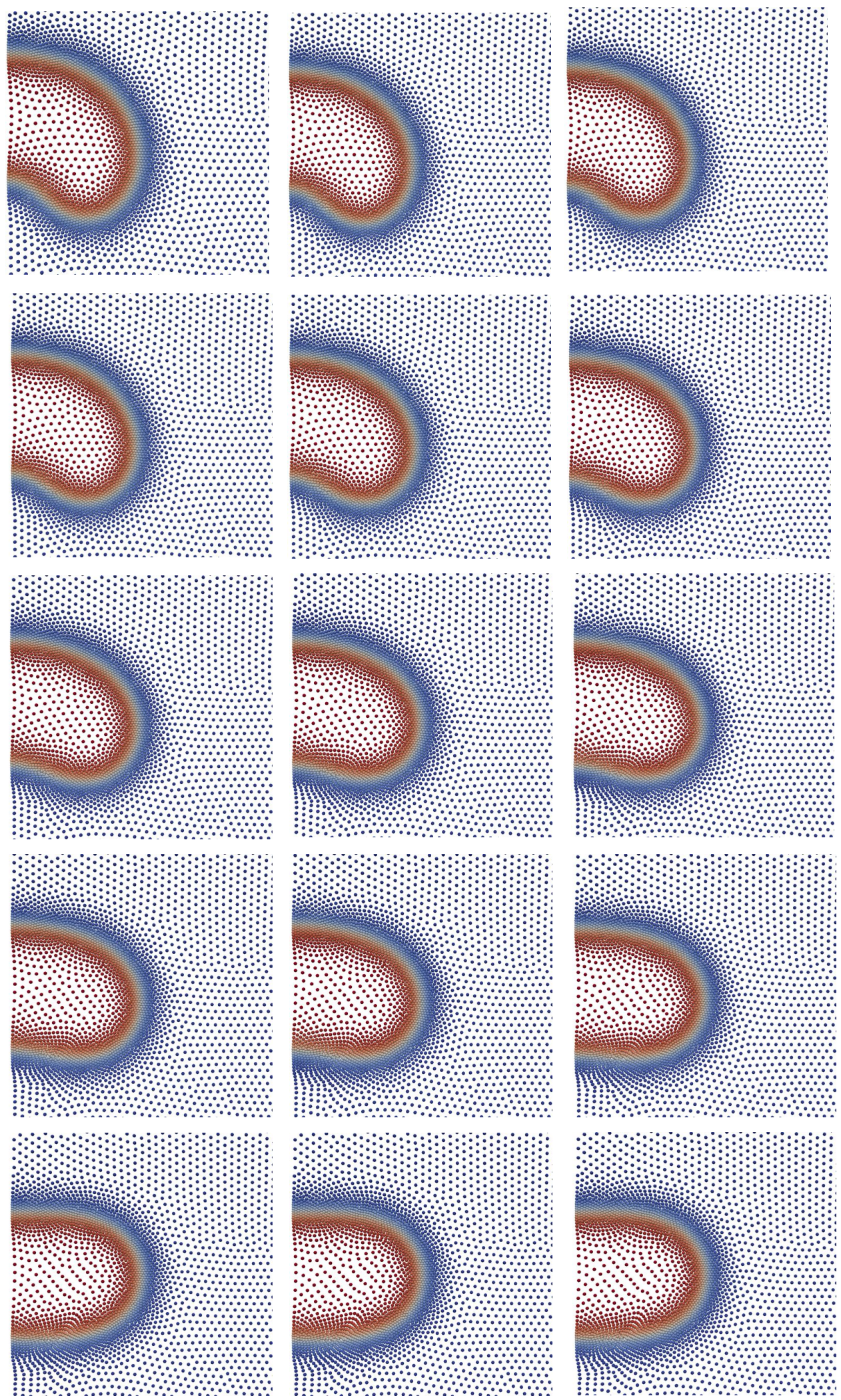

Figure 3: Relaxation dynamics of an oblate vesicle in a viscous fluid, initially brought out-of-equilibrium. We represent the time-evolution of the nodes $y^{a}(t)$, color-coded with the phase-field. The adapted grid has 6124 nodes. 


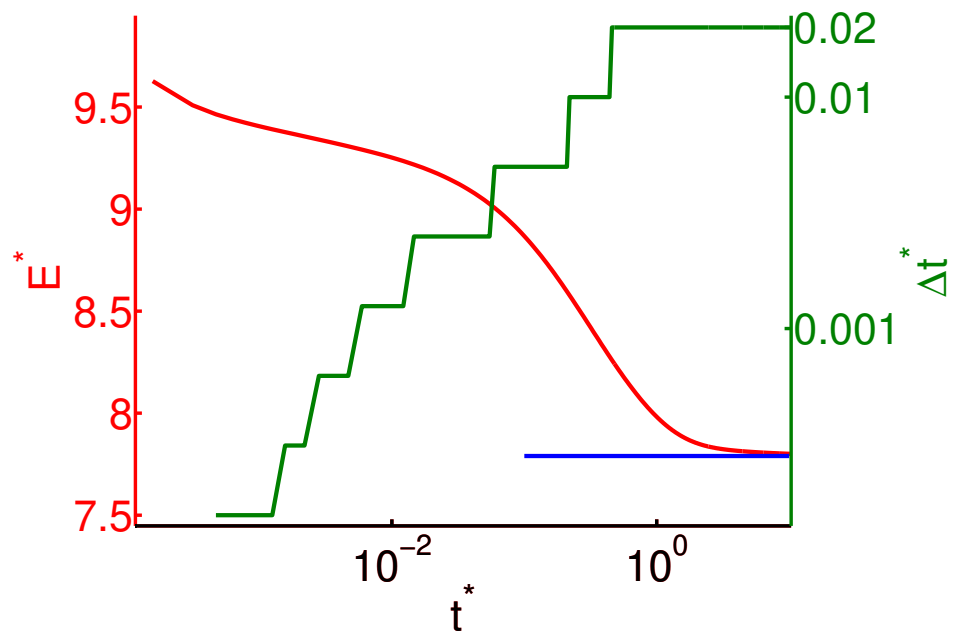

Figure 4: Energy relaxation and time adaptive strategy for the dynamics depicted in Fig. 3. Energy and time-step evolution, where time is represented in logarithmic scale. The blue horizontal line shows the equilibrium energy obtained independently with a parametric method based on B-Splines. 

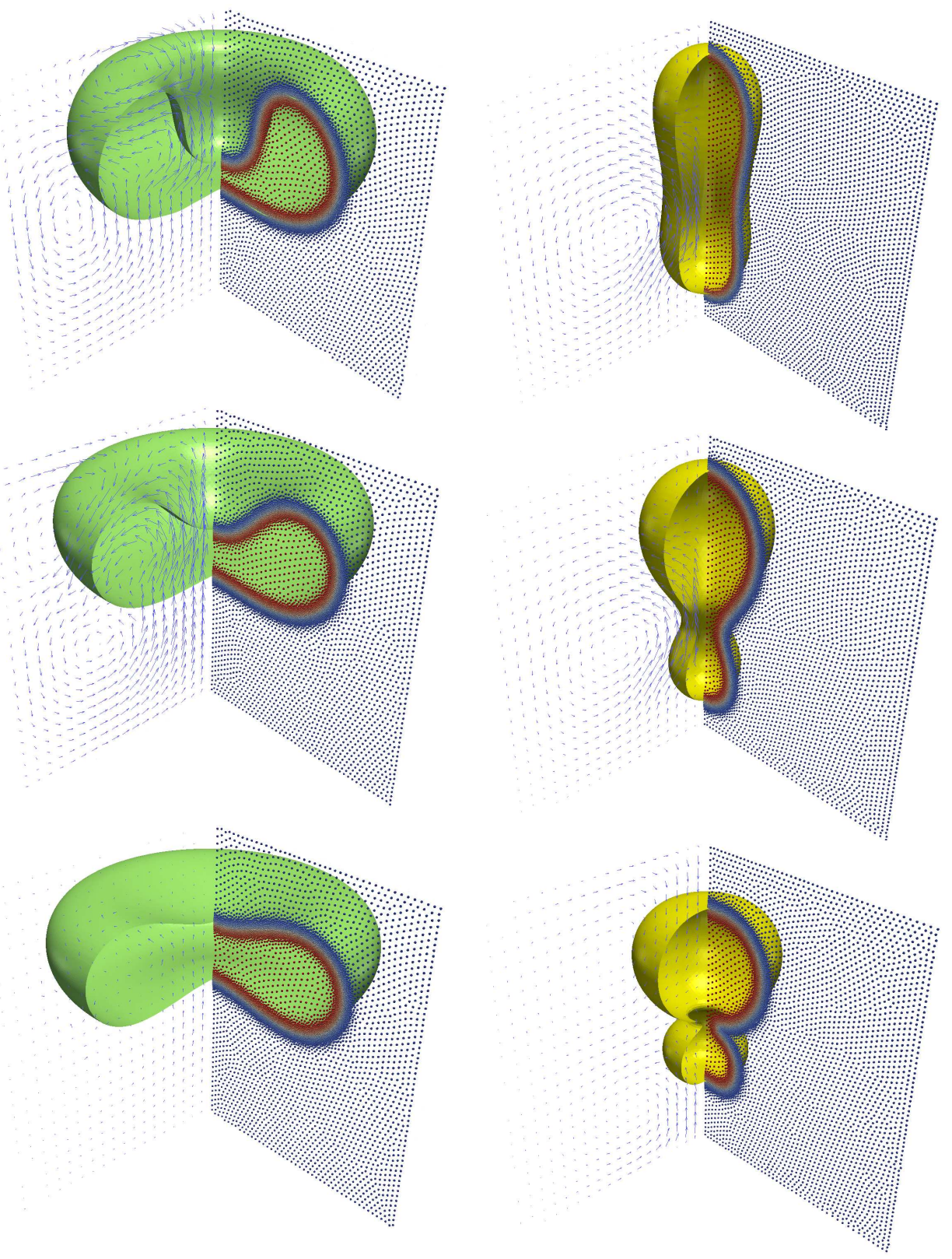

Figure 5: Left: Stomacyte-discocyte transition. Right: Prolate vesicle evolving after an instantaneous change of spontaneous curvature (6,124 nodes, constant area and volume). The points represent the nodes, color-coded with the phase-field, while the arrows depict the flow field in a symmetry plane. 

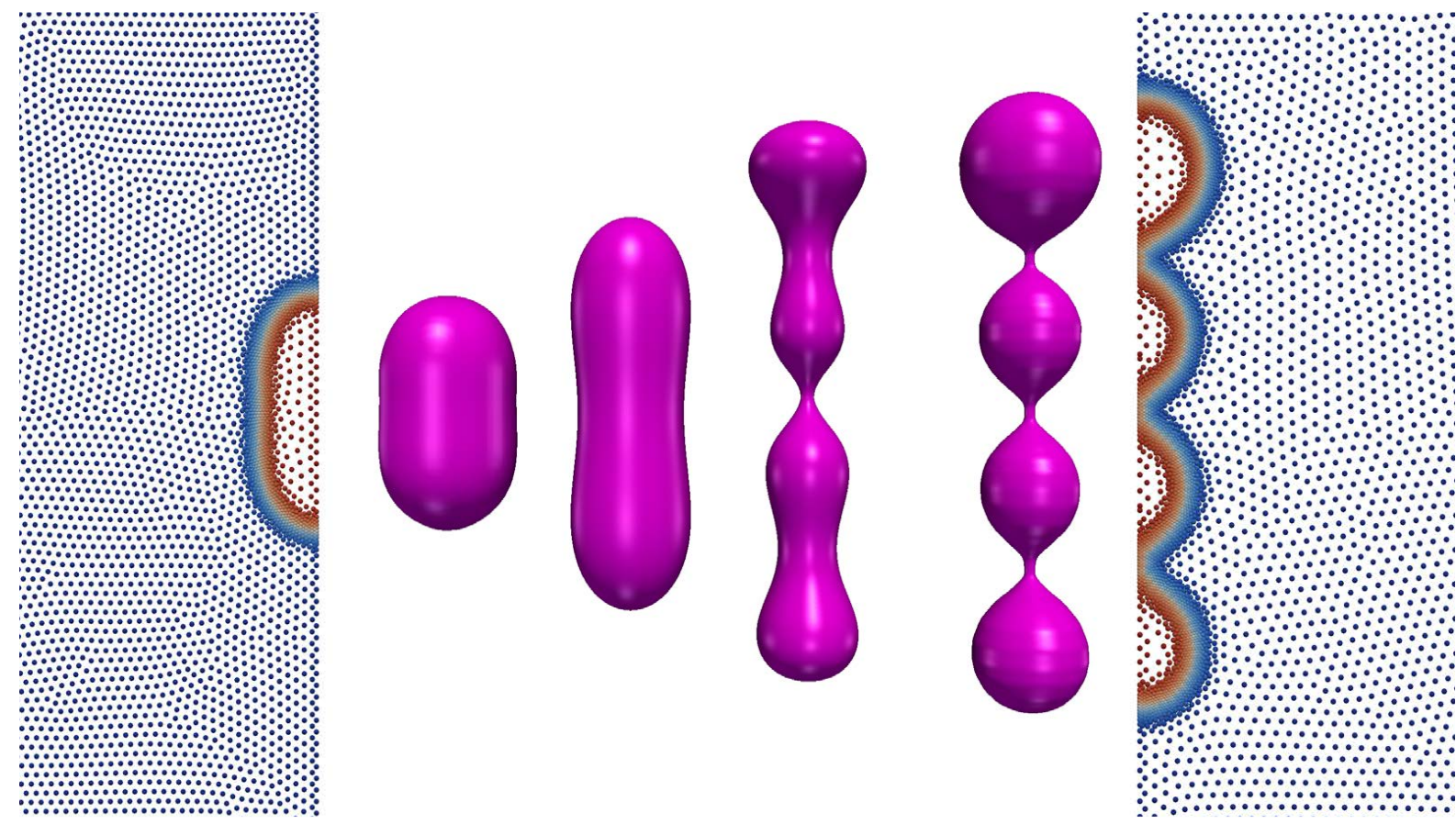

Figure 6: Relaxation dynamics of a constant area vesicle under combined volume decrease and spontaneous curvature increase $(12,650$ nodes, constant area). 

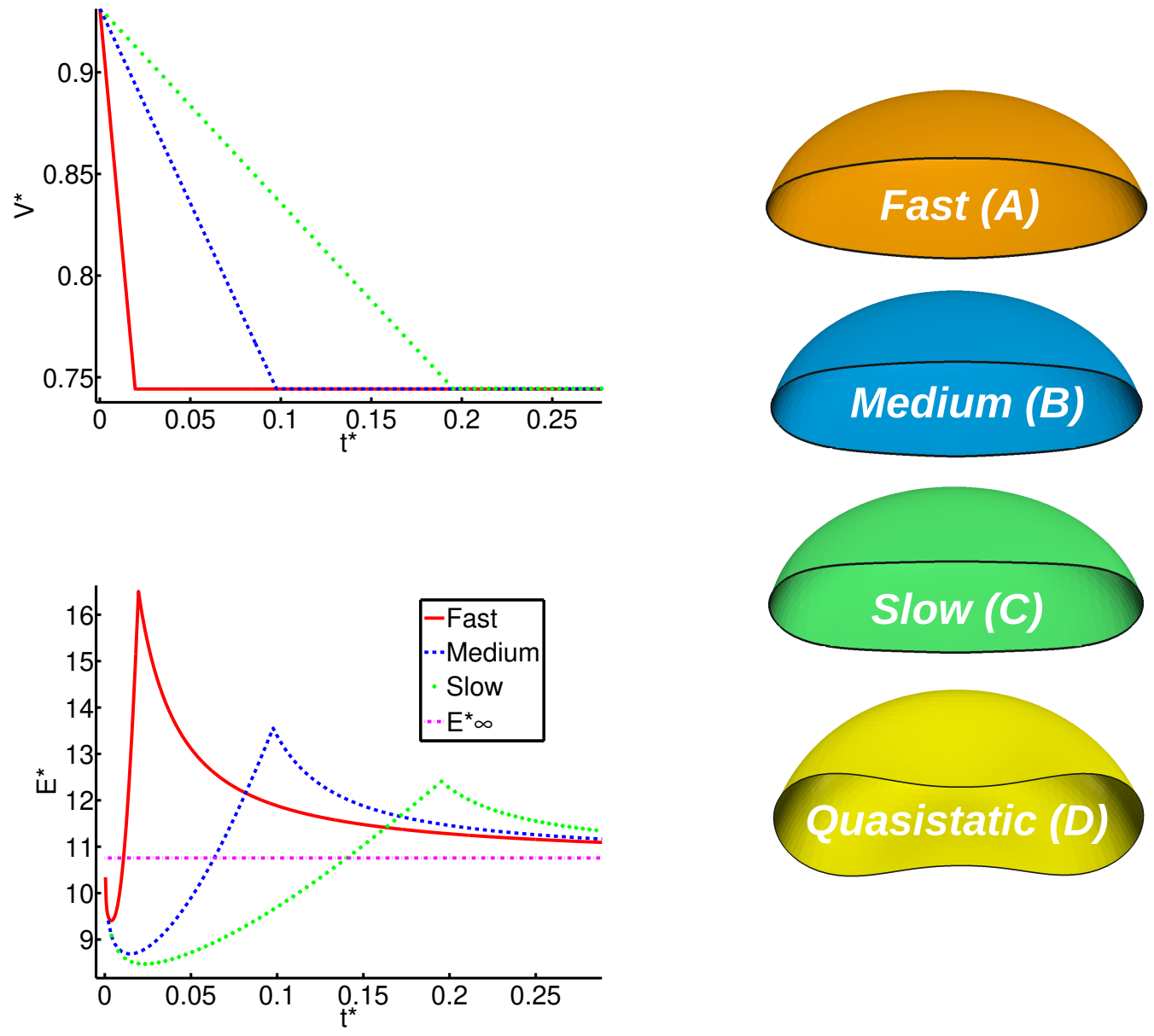

Figure 7: Kinetic effects. Top-left: Enclosed volume evolution for the three volume decrease rates considered. Bottom-left: Energy evolution for the three rates. Right: Shapes at the end of the volume reduction, therefore enclosing the same volume, and equilibrium shape (D) for this enclosed volume. $(6,124$ nodes, constant area). 